Original Paper

\title{
Rotordynamic Instabilities Caused by the Fluid Force Moments on the Backshroud of a Francis Turbine Runner
}

\author{
Bingwei Song ${ }^{1,2}$, Hironori Horiguchi ${ }^{2}$, Zhenyue Ma $^{1}$, Yoshinobu Tsujimoto ${ }^{2}$ \\ ${ }^{1}$ Graduate School of Hydraulic Engineering, Dalian University of Technology \\ No.2 Linggong Road, Ganjingzi, Dalian, 116024, China \\ ${ }^{2}$ Graduate School of Engineering Science, Osaka University \\ 1-3 Machikaneyama, Toyonaka, Osaka, 560-8531, Japan
}

\begin{abstract}
Severe flexural vibration of the rotor shaft of a Francis turbine runner was experienced in the past. It was shown that the vibration was caused by the fluid forces and moments on the backshroud of the runner associated with the leakage flow through the back chamber. The aim of the present paper is to study the self-excited rotor vibration caused by the fluid force moments on the backshroud of a Francis turbine runner. The rotor vibration includes two fundamental motions, one is a whirling motion which only has a linear displacement and the other is a precession motion which only has an angular displacement. Accordingly, two types of fluid force moment are exerted on the rotor, the moment due to whirl and the moment due to precession. The main focus of the present paper is to clarify the contribution of each moment to the self-excited vibration of an overhung rotor. The runner was modeled by a disk and the whirl and the precession moments on the backshroud of the runner caused by the leakage flow were evaluated from the results of model tests conducted before. A lumped parameter model of a cantilevered rotor was used for the vibration analysis. By examining the frequency, the damping rate, the amplitude ratio of lateral and angular displacements for the cases with longer and shorter overhung rotor, it was found that the precession moment is more important for smaller overhung rotors and the whirl moment is more important for larger overhung rotors, although both types of moment due to the leakage flow can cause self-excited vibration of an overhung rotor.
\end{abstract}

Keywords: Rotordynamic instabilities, fluid force moments, Francis turbine runner, backshroud

\section{Introduction}

In turbomachinery, fluid-induced vibrations are typical self-excited vibrations which absorb many prior researchers' attentions. The rotordynamic instability of rotor systems caused by the fluid forces and moments acting on the shroud of the pump impeller were studied by many researchers. For the front shroud, the rotordynamic characteristics and the rotordynamic fluid forces caused by the leakage flows were examined experimentally [1,2], and the effect of swirl on the fluid forces was investigated in [3]. For the backshroud, the experimental results of fluid moment on a centrifugal impeller shroud in precessing motion were discussed based on the bulk flow model to elucidate the fundamental flow mechanism [4]. The flow in backshroud/casing clearance of precessing centrifugal impeller was measured in [5].

A severe flexural vibration of a rotor shaft of a Francis turbine generator shown in Fig. 1 was experienced when the load exceeded a certain value $[6,7]$. It was shown that the vibration was self-excited by the fluid forces and moments acting on the backshroud of the runner associated with the leakage flow through the back chamber.

The motion of a rotor can be assorted to two fundamental modes. One is whirling motion which only has a lateral linear displacement $|\varepsilon|$, and the other is precession motion which only has an angular displacement $|\alpha|$.

Reference [1-3] discussed the rotordynamic instability caused by the rotordynamic fluid forces under whirling motion associated with the leakage flow. On the other hand, $[4,5]$ discussed the instability in precession motion. Since the whirling and precessing modes occur simultaneously, Kanemori and Iwatsubo [8,9] studied the rotordynamic forces and moments on a long seal under whirling and precessing vibrations. They provided a complete set of rotordynamic force and moment coefficients needed for the vibration analysis. However, further rotordynamic analysis and the effect of the fluid force moments caused by the whirling and precession motions on the stability were not specially discussed.

Received March 18 2010; accepted for publication March 24 2010: Review conducted by Prof. Tong Seop Kim. (Paper number O10005K) Corresponding author: Bingwei Song, bingweisong@dl.cn 


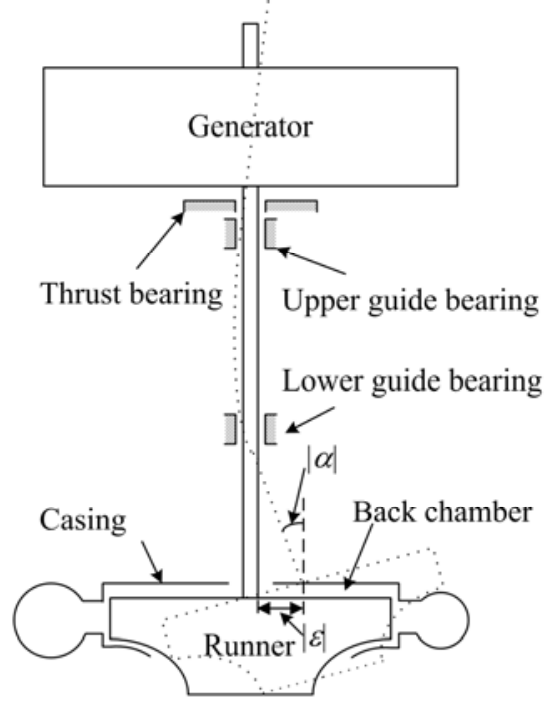

Fig. 1 Flexural vibration of a Francis turbine

In order to study the rotordynamic problem of the Francis turbine runner as reported in [6], the authors have measured the rotordynamic force moments on a whirling and precessing disk simulating the backshroud of a Francis turbine runner, respectively $[10,11]$. In [10], it was shown by a simplified vibration analysis that the moment on the rotor caused by the whirl can cause whirl instability through structural coupling for the case of an overhung rotor. The purpose of the present study is to verify this result with a more elaborate vibration analysis including both whirl and precession modes. In addition, the relative importance of whirl and precession moments is examined. The rotordynamic moment coefficients were determined from the fluid force moments measured in [10] and [11]. The stability analysis of a cantilevered rotor is carried out by using a lumped parameter model. By comparing the vibration modes of the rotor in the cases with "whirl", "precession", and "whirl and precession" moments, it was shown that the precession moment has a predominant effect for the stability of a shorter shaft. On the other hand, the whirl moment has a predominant effect for the stability of a longer shaft.

\section{Fluid Force Measurements}

\subsection{Outline of Experiments}

Figure 2 shows the coordinate system and the definitions of the fluid force moments in whirling and precession motions. Figure 2(a) shows the general case of overhung rotor vibration. The rotor rotates with an angular velocity of $\omega$ and whirls/precesses with an angular velocity of $\Omega$. The motion of the rotor can be decomposed to the whirling motion of Fig. 2(b) and precession motion of Fig. 2(c). The definitions of the fluid force moments in whirling and precession motions are shown in Figs. 2(b) and (c), respectively. For the whirling motion, the disk has only a linear displacement $\varepsilon$. The normal moment $M_{n-w}$ is a component normal to the whirl obit and the tangential moment $M_{t_{-} W}$ is a component tangential to it. For the precession motion, the disk has only an angular displacement $\alpha$. The tangential component $M_{t_{-} P}$ is defined as the component in the angular displacement vector and the normal component $M_{n_{-} P}$ is normal to it.

The schematic of the experimental facility is shown in Fig. 3. The Francis turbine runner is modeled by a disk with the diameter of $D_{T}=299 \mathrm{~mm}$ set close to the casing with the axial clearance $C_{2}$. The radial clearance $C_{1}$ models the seal at the outer periphery. The disk rotates with an angular velocity of $\omega=400 \mathrm{rpm}$. The rotational tip velocity $U_{T}\left(=\left(D_{T} / 2\right) \omega\right)$ is used as the representative velocity. A swirl generator is used to produce the inlet swirl. The working fluid is water. The radius of the disk $R_{T}=D_{T} / 2$ is $149.5 \mathrm{~mm}$. The inner radius of the casing $R_{\text {inner }}$ is $47.5 \mathrm{~mm}$. The mean radial clearance is $C_{1}=1 \mathrm{~mm}$. The mean axial clearance $C_{2}$ can be adjusted to $2 \mathrm{~mm}, 4 \mathrm{~mm}$, and $6 \mathrm{~mm}$. The leakage flow is produced by an external pump. The leakage flow rate is represented by the nominal flow velocity $v_{l}$ in the radial clearance $C_{l}$. The inlet swirl velocity was represented by the nominal

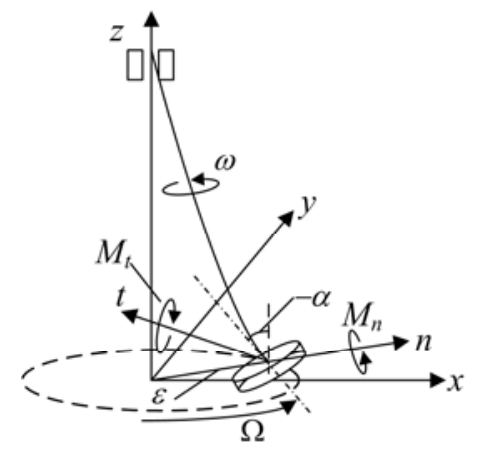

(a) Whirling and Precession motions

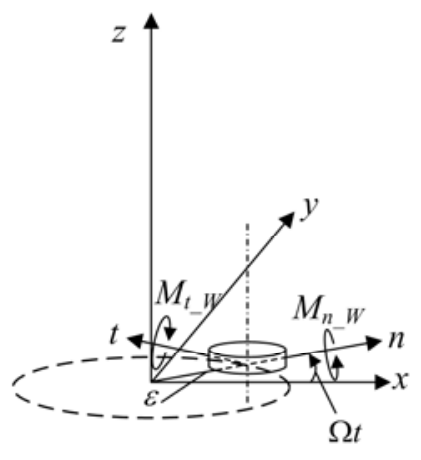

(b) Whirling motion

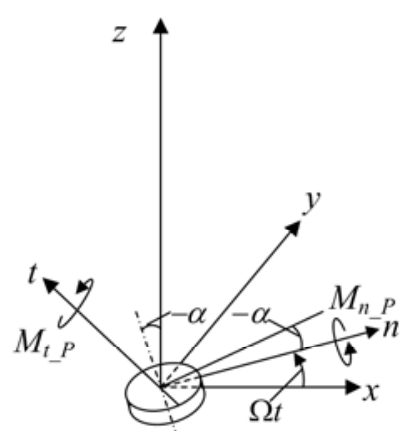

(c) Precession motion

Fig. 2 Coordinate system 


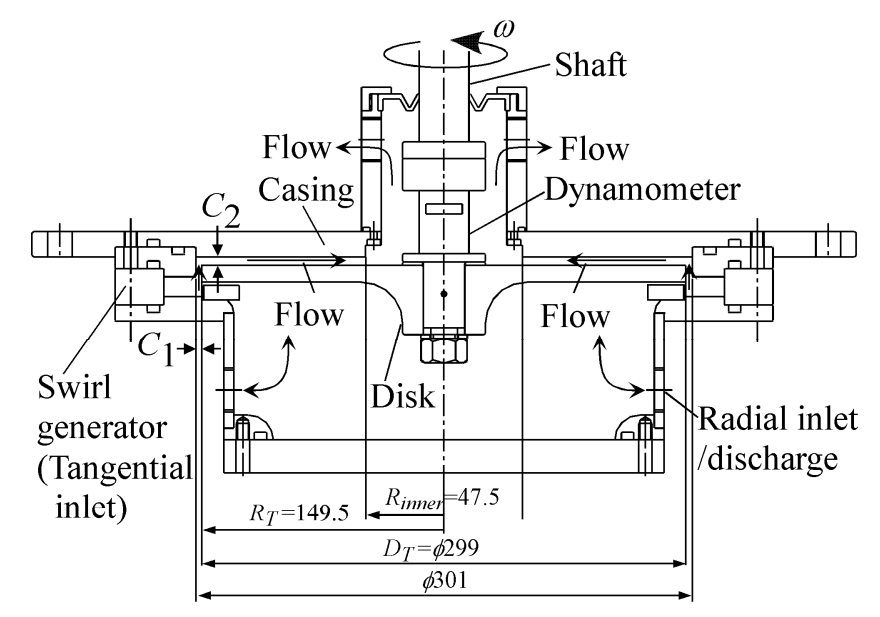

Fig. 3 Schematic of experimental facility

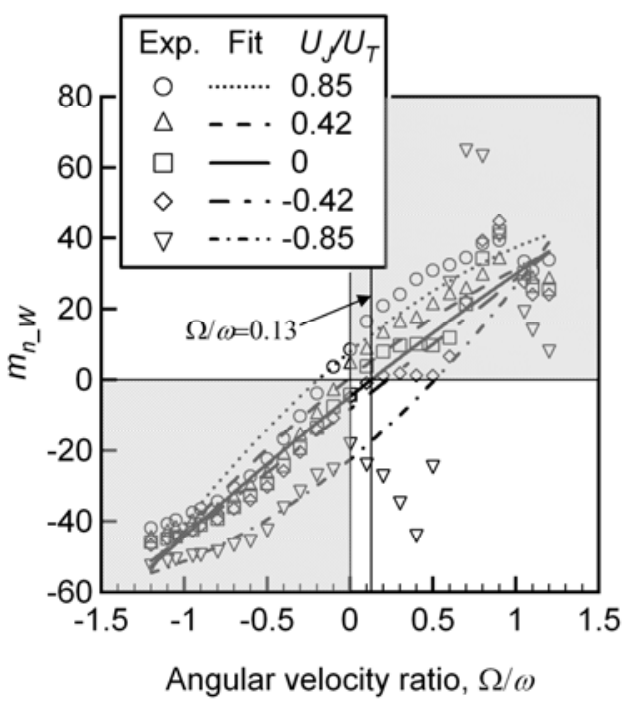

(a) Normal moment

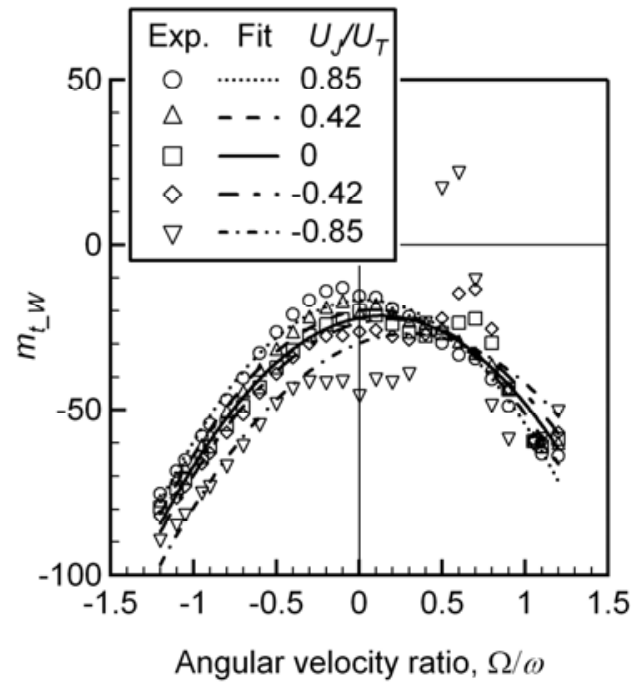

(b) Tangential moment

Fig. 4 Fluid force moments at various pre-swirl velocities in the case of $C_{2}=4 \mathrm{~mm}$ and $v_{l} / U_{T}=0.170$ under whirling motion

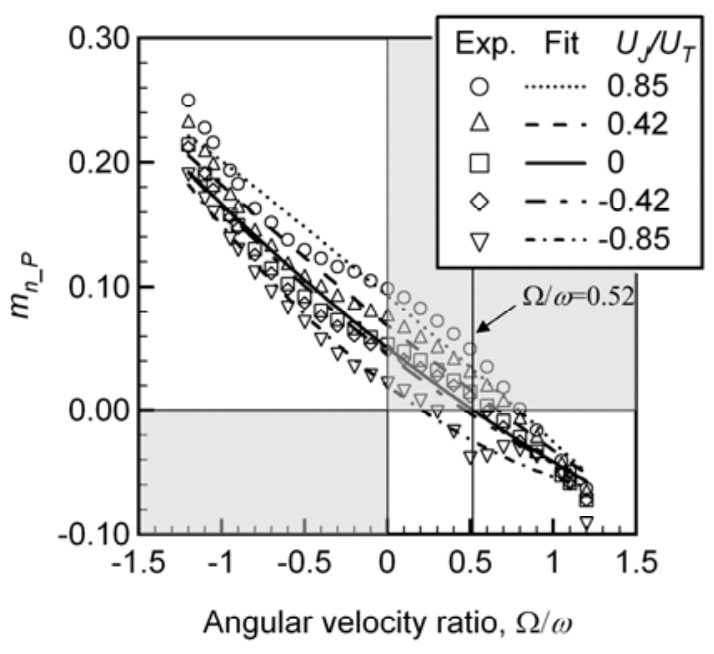

(a) Normal moment

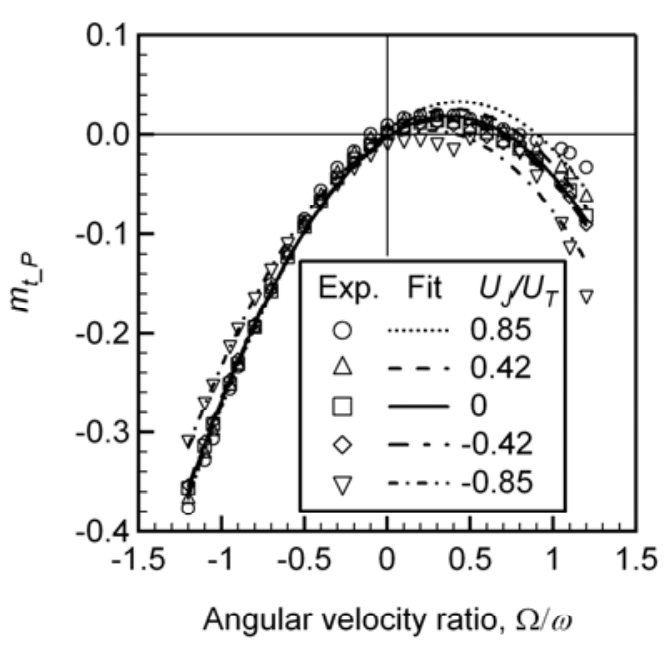

(b) Tangential moment

Fig. 5 Fluid force moments at various pre-swirl velocities in the case of $C_{2}=4 \mathrm{~mm}$ and $v_{l} / U_{T}=0.170$ under precession motion

(tangential) velocity $U_{J}$ through the swirl generator. The radial inlet/discharge was used to adjust the leakage flow rate.

In the cases of whirling or precession motion, a linear displacement $|\varepsilon|(=0.5 \mathrm{~mm})$ or an angular displacement $|\alpha|(=0.48 \mathrm{deg})$ were given. The angular velocity of whirl and precession frequency is represented by $\Omega$. The angular velocity ratio $\Omega / \omega$ was varied from -1.2 to 1.2. For the detailed experimental facility and the measurement of the fluid force moments, refer to [10, 11]. 


\subsection{Experimental Fluid Force Moments}

Figure 4 shows the normalized whirl moments $m_{n_{-} W} \equiv M_{n_{-} W} /\left(\rho \pi R_{T}^{3} C_{2} \omega^{2}|\varepsilon|\right), m_{t_{-} W} \equiv M_{t_{-} W} /\left(\rho \pi R_{T}^{3} C_{2} \omega^{2}|\varepsilon|\right)$ at various preswirl velocities $U_{J} / U_{T}$ in the case of $C_{2}=4 \mathrm{~mm}$ and $v_{l} / U_{T}=0.170$, where $U_{J}$ is the tangential jet velocity from the swirl generator shown in Fig. 3 and defined positive when the swirl direction is the same as disk rotation. It was shown by a simplified vibration model [7] that the normal moment destabilizes the whirling or precession motion when it has the same sign as the angular velocity $\Omega$ of whirling or precession motion. The destabilizing regions are colored gray in Figs. 4(a) and 5(a). The result in Fig. 4(a) shows that the normal moment $m_{n \_}$destabilizes the whirling motion in a large region of angular velocity ratio $\Omega / \omega$.

Figure 5 shows the normalized precession moments $m_{n_{-} P} \equiv M_{n_{-} P} /\left(/\left(\rho R_{T}^{6} \omega^{2}|\alpha| / C_{2}\right)\right.$ and $m_{t_{-} P} \equiv M_{t_{-} P} P /\left(\rho R_{T}^{6} \omega^{2}|\alpha| / C_{2}\right)$. It shows that the normal moment $m_{n_{-} P}$ encourages the precession motion at small forward precession angular velocity ratios as shown in Fig. $5(\mathrm{a})$.

\section{Stability Analysis}

\subsection{Matrix Representation of Fluid Force Moment}

For the vibration analysis, it is conventional to represent the fluid forces $\left(F_{x}, F_{y}\right)_{f}$ and moments $\left(M_{x}, M_{y}\right)_{f}$ in terms of the linear displacements $(X, Y)$ and angular displacements $\left(A_{x}, A_{y}\right)$ as follows [12].

$$
\begin{aligned}
& -\left\{\begin{array}{l}
F_{x} \\
F_{y} \\
M_{y} \\
M_{x}
\end{array}\right\}=\left\{\begin{array}{cccc}
K & k & -K_{\varepsilon \alpha} & k_{\varepsilon \alpha} \\
-k & K & k_{\varepsilon \alpha} & K_{\varepsilon \alpha} \\
-K_{\alpha \varepsilon} & -k_{\alpha \varepsilon} & K_{\alpha} & -k_{\alpha} \\
-k_{\alpha \varepsilon} & K_{\alpha \varepsilon} & k_{\alpha} & K_{\alpha}
\end{array}\right\}\left\{\begin{array}{l}
X \\
Y \\
A_{y} \\
A_{x}
\end{array}\right\} \\
& +\left\{\begin{array}{cccc}
C & c & -C_{\varepsilon \alpha} & C_{\varepsilon \alpha} \\
-C & C & C_{\varepsilon \alpha} & C_{\varepsilon \alpha} \\
-C_{\alpha \varepsilon} & -C_{\alpha \varepsilon} & C_{\alpha} & -C_{\alpha} \\
-C_{\alpha \varepsilon} & C_{\alpha \varepsilon} & C_{\alpha} & C_{\alpha}
\end{array}\right\}\left\{\begin{array}{l}
\dot{X} \\
\dot{Y} \\
\dot{A}_{y} \\
\dot{A}_{x}
\end{array}\right\}+\left\{\begin{array}{cccc}
M & m & -M_{\varepsilon \alpha} & m_{\varepsilon \alpha} \\
-m & M & m_{\varepsilon \alpha} & M_{\varepsilon \alpha} \\
-M_{\alpha \varepsilon} & -m_{\alpha \varepsilon} & M_{\alpha} & -m_{\alpha} \\
-m_{\alpha \varepsilon} & M_{\alpha \varepsilon} & m_{\alpha} & M_{\alpha}
\end{array}\right\}\left\{\begin{array}{l}
\ddot{X} \\
\ddot{Y} \\
\ddot{A}_{y} \\
\ddot{A}_{x}
\end{array}\right\}
\end{aligned}
$$

In the present study to clarify the contribution of the moments, we neglect the fluid forces. The elements of the coefficient matrix can be obtained by fitting the normal and tangential moments by parabolic curves of Eqs. (2) and (3) [12].

For the whirling motion,

$$
\left\{\begin{array}{l}
m_{n_{-} W}=\tilde{k}_{\alpha \varepsilon}-\tilde{C}_{\alpha \varepsilon} f-\tilde{m}_{\alpha \varepsilon} f^{2} \\
m_{t_{-} W}=\tilde{K}_{\alpha \varepsilon}+\tilde{c}_{\alpha \varepsilon} f-\tilde{M}_{\alpha \varepsilon} f^{2}
\end{array}\right.
$$
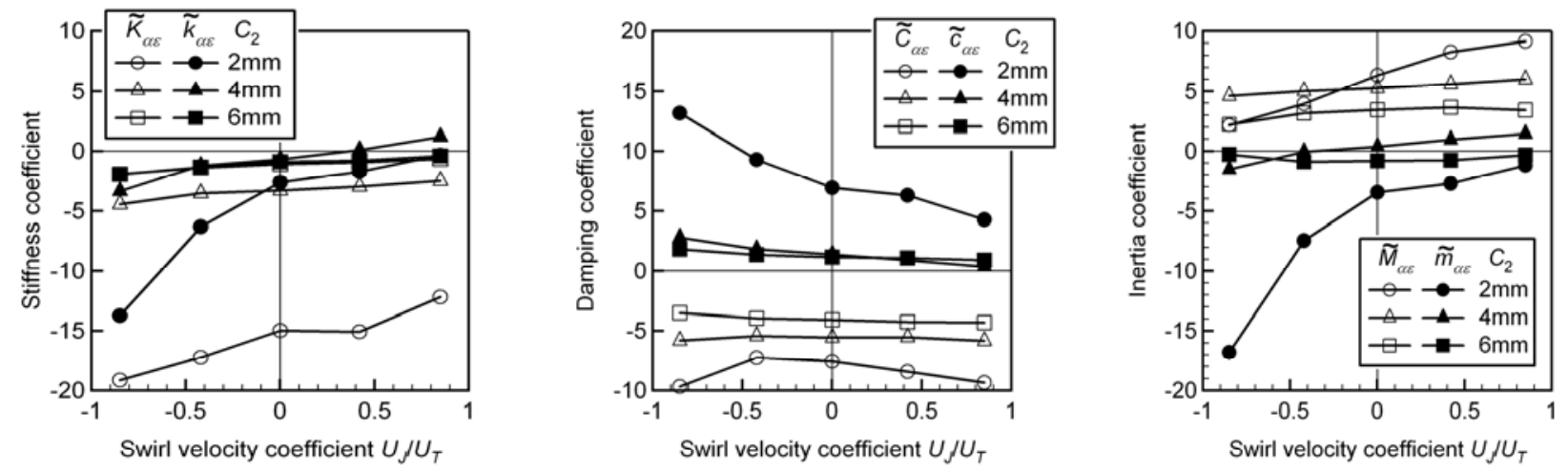

Fig. 6 Swirl effect on rotordynamic coefficients obtained from the fluid force moments under whirling motion
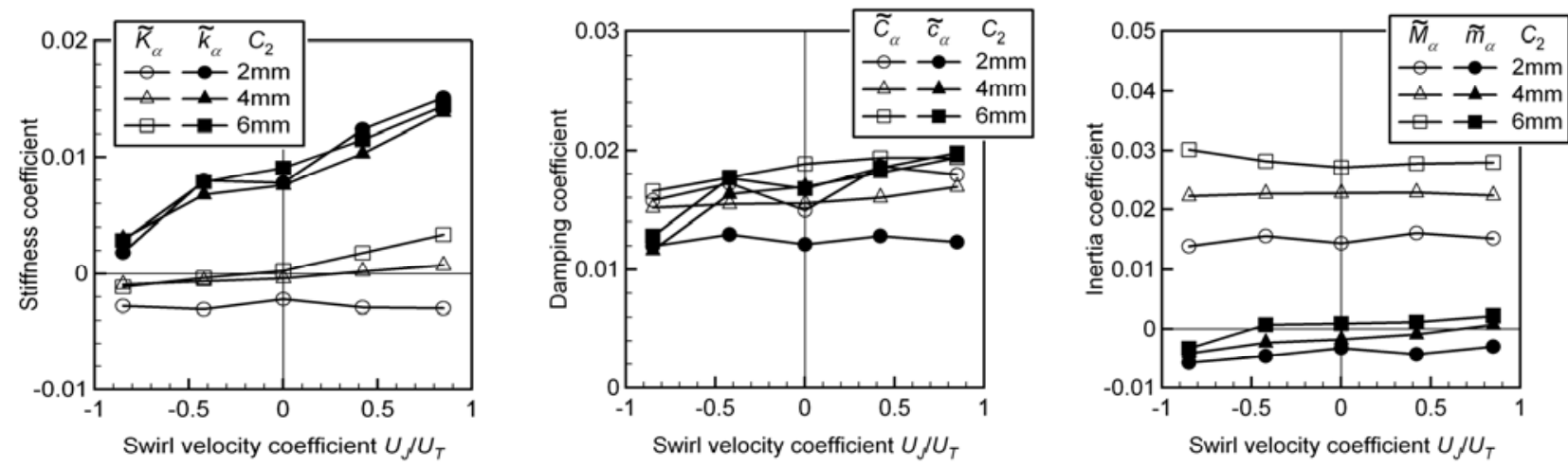

Fig. 7 Swirl effect on rotordynamic coefficients obtained from the fluid force moments under precession motion 
For the precession motion,

$$
\left\{\begin{array}{l}
m_{n_{-} P}=\tilde{k}_{\alpha}-\tilde{C}_{\alpha} f-\tilde{m}_{\alpha} f^{2} \\
m_{t_{-} P}=\tilde{K}_{\alpha}+\tilde{C}_{\alpha} f-\tilde{M}_{\alpha} f^{2}
\end{array}\right.
$$

where, the matrix elements are normalized as follows.

$$
\begin{aligned}
& \tilde{K}_{\alpha \varepsilon}=K_{\alpha \varepsilon} /\left(\rho \pi R_{T}^{3} C_{2} \omega^{2}\right), \tilde{k}_{\alpha \varepsilon}=k_{\alpha \varepsilon} /\left(\rho \pi R_{T}^{3} C_{2} \omega^{2}\right), \tilde{C}_{\alpha \varepsilon}=C_{\alpha \varepsilon} /\left(\rho \pi R_{T}^{3} C_{2} \omega\right), \tilde{c}_{\alpha \varepsilon}=c_{\alpha \varepsilon} /\left(\rho \pi R_{T}^{3} C_{2} \omega\right), \tilde{M}_{\alpha \varepsilon}=M_{\alpha \varepsilon} /\left(\rho \pi R_{T}^{3} C_{2}\right), \\
& \tilde{m}_{\alpha \varepsilon}=m_{\alpha \varepsilon} /\left(\rho \pi R_{T}^{3} C_{2}\right), \\
& \tilde{K}_{\alpha}=K_{\alpha} /\left(\rho R_{T}^{6} \omega^{2} / C_{2}\right), \tilde{k}_{\alpha}=k_{\alpha} /\left(\rho R_{T}^{6} \omega^{2} / C_{2}\right), \tilde{C}_{\alpha}=C_{\alpha} /\left(\rho R_{T}^{6} \omega / C_{2}\right), \tilde{c}_{\alpha}=c_{\alpha} /\left(\rho R_{T}^{6} \omega / C_{2}\right), \\
& \tilde{M}_{\alpha}=M_{\alpha} /\left(\rho R_{T}^{6} / C_{2}\right), \tilde{m}_{\alpha}=m_{\alpha} /\left(\rho R_{T}^{6} / C_{2}\right), f=\Omega / \omega,
\end{aligned}
$$

The nondimensional rotordynamic coefficients, which are obtained by curve fitting the experimental data shown in Figs. 4 and 5 , are plotted against the swirl velocity coefficient $U_{J} / U_{T}$ in Figs. 6 and 7 for various values of $C_{2}$. The results are used in the calculations in the following sections.

\subsection{Lumped Parameter Model}

For a cantilevered rotor system shown in Fig. 2, the equations of the motion of the rotor can be represented [13] by

$$
\left[\begin{array}{cccc}
M_{d} & 0 & 0 & 0 \\
0 & M_{d} & 0 & 0 \\
0 & 0 & I_{d} & 0 \\
0 & 0 & 0 & I_{d}
\end{array}\right]\left\{\begin{array}{l}
\ddot{X} \\
\ddot{Y} \\
\ddot{A}_{y} \\
\ddot{A}_{x}
\end{array}\right\}+\omega\left[\begin{array}{cccc}
0 & 0 & 0 & 0 \\
0 & 0 & 0 & 0 \\
0 & 0 & 0 & -I_{p} \\
0 & 0 & I_{p} & 0
\end{array}\right]\left\{\begin{array}{l}
\dot{X} \\
\dot{Y} \\
\dot{A}_{y} \\
\dot{A}_{x}
\end{array}\right\}+\left[\begin{array}{cccc}
K_{11} & 0 & K_{12} & 0 \\
0 & K_{11} & 0 & -K_{12} \\
K_{21} & 0 & K_{22} & 0 \\
0 & -K_{21} & 0 & K_{22}
\end{array}\right]\left\{\begin{array}{l}
X \\
Y \\
A_{y} \\
A_{x}
\end{array}\right\}=\left\{\begin{array}{l}
F_{x} \\
F_{y} \\
M_{y} \\
M_{x}
\end{array}\right\}_{f}
$$

where, $\left\{F_{x}, F_{y}, M_{x}, M_{y}\right\}_{f}$ are fluid forces and moments on the rotor (Although we will neglect the fluid forces, we retain them in the development of equations.). The stiffness matrix of the shaft with the overhung length $L$ can be represented as follows.

$$
[\mathbf{K}]=\left[\begin{array}{cccc}
K_{11} & 0 & K_{12} & 0 \\
0 & K_{11} & 0 & -K_{12} \\
K_{21} & 0 & K_{22} & 0 \\
0 & -K_{21} & 0 & K_{22}
\end{array}\right]=\left[\begin{array}{cccc}
12 E I / L^{3} & 0 & 6 E I / L^{2} & 0 \\
0 & 12 E I / L^{3} & 0 & -6 E I / L^{2} \\
6 E I / L^{2} & 0 & 4 E I / L & 0 \\
0 & -6 E I / L^{2} & 0 & 4 E I / L
\end{array}\right]
$$

If we use the complex parameters defined as follows,

$$
\varepsilon=X+j Y, A=A_{x}+j A_{y}, \quad F_{f}=F_{x f}+j F_{y f} ; M_{f}=M_{x f}+j M_{y f}
$$

equation (4) can be simplified as below.

$$
\left\{\begin{array}{l}
M_{d} \ddot{\varepsilon}+K_{11} \varepsilon-j K_{12} A=F_{f} \\
I_{d} \ddot{A}+K_{22} A+j K_{21} \varepsilon=M_{f}+M_{G}
\end{array}\right.
$$

where $M_{G}=j \omega I_{p} \dot{A}$ is the gyroscopic moment. The fluid forces and moments include the fluid forces and moments due to both the whirling and precession motions as expressed below.

$$
\begin{aligned}
& F_{f}=F_{f_{-} W}+F_{f_{-} P} \\
& M_{f}=M_{f_{-} W}+M_{f_{-} P}
\end{aligned}
$$

For the whirling motion,

$$
-\left\{\begin{array}{l}
F_{x} \\
F_{y} \\
M_{y} \\
M_{x}
\end{array}\right\}_{f_{-} W}=\left\{\begin{array}{cc}
K & k \\
-k & K \\
-K_{\alpha \varepsilon} & -k_{\alpha \varepsilon} \\
-k_{\alpha \varepsilon} & K_{\alpha \varepsilon}
\end{array}\right\}\left\{\begin{array}{l}
X \\
Y
\end{array}\right\}+\left\{\begin{array}{cc}
C & C \\
-C & C \\
-C_{\alpha \varepsilon} & -C_{\alpha \varepsilon} \\
-C_{\alpha \varepsilon} & C_{\alpha \varepsilon}
\end{array}\right\}\left\{\begin{array}{l}
\dot{X} \\
\dot{Y}
\end{array}\right\}\left\{\begin{array}{cc}
M & m \\
-m & M \\
-M_{\alpha \varepsilon} & -m_{\alpha \varepsilon} \\
-m_{\alpha \varepsilon} & M_{\alpha \varepsilon}
\end{array}\right\}\left\{\begin{array}{l}
\ddot{X} \\
\ddot{Y}
\end{array}\right\}
$$

The complex expression of Eq. (10) can be stated

$$
\left\{\begin{array}{l}
F_{f_{-} W}=-(K-j k) \varepsilon-(C-j c) \dot{\varepsilon}-(M-j m) \ddot{\varepsilon} \\
M_{f_{-} W}=\left(k_{\alpha \varepsilon}+j K_{\alpha \varepsilon}\right) \varepsilon+\left(c_{\alpha \varepsilon}+j C_{\alpha \varepsilon}\right) \dot{\varepsilon}+\left(m_{\alpha \varepsilon}+j M_{\alpha \varepsilon}\right) \ddot{\varepsilon}
\end{array}\right.
$$

For the precession motion,

$$
-\left\{\begin{array}{l}
F_{x} \\
F_{y} \\
M_{y} \\
M_{x}
\end{array}\right\}=\left\{\begin{array}{cc}
-K_{\varepsilon \alpha} & k_{\varepsilon \alpha} \\
k_{\varepsilon \alpha} & K_{\varepsilon \alpha} \\
K_{\alpha} & -k_{\alpha} \\
k_{\alpha} & K_{\alpha}
\end{array}\right\}\left\{\begin{array}{c}
A_{y} \\
A_{x}
\end{array}\right\}+\left\{\begin{array}{cc}
-C_{\varepsilon \alpha} & c_{\varepsilon \alpha} \\
C_{\varepsilon \alpha} & C_{\varepsilon \alpha} \\
C_{\alpha} & -C_{\alpha} \\
c_{\alpha} & C_{\alpha}
\end{array}\right\}\left\{\begin{array}{c}
\dot{A}_{y} \\
\dot{A}_{x}
\end{array}\right\}+\left\{\begin{array}{cc}
-M_{\varepsilon \alpha} & m_{\varepsilon \alpha} \\
k_{\varepsilon \alpha} & M_{\varepsilon \alpha} \\
M_{\alpha} & -m_{\alpha} \\
m_{\alpha} & M_{\alpha}
\end{array}\right\}\left\{\begin{array}{l}
\ddot{A}_{y} \\
\ddot{A}_{x}
\end{array}\right\}
$$

The complex expression of Eq. (12) can be stated

$$
\left\{\begin{array}{l}
F_{f_{-} P}=-\left(k_{\varepsilon \alpha}+j K_{\varepsilon \alpha}\right) A-\left(c_{\varepsilon \alpha}+j C_{\varepsilon \alpha}\right) \dot{A}-\left(m_{\varepsilon \alpha}+j M_{\varepsilon \alpha}\right) \ddot{A} \\
M_{f_{-} P}=-\left(K_{\alpha}-j k_{\alpha}\right) A-\left(C_{\alpha}-j c_{\alpha}\right) \dot{A}-\left(M_{\alpha}-j m_{\alpha}\right) \ddot{A}
\end{array}\right.
$$


Substituting Eqs. (8), (9), (11), (13) into Eq. (7), we can get

$$
\begin{aligned}
& \left(M_{d}+M-j m\right) \ddot{\varepsilon}+(C-j c) \dot{\varepsilon}+\left(K_{11}+K-j k\right) \varepsilon+\left(k_{\varepsilon \alpha}+j K_{\varepsilon \alpha}-j K_{12}\right) A+\left(c_{\varepsilon \alpha}+j C_{\varepsilon \alpha}\right) \dot{A}+\left(m_{\varepsilon \alpha}+j M_{\varepsilon \alpha}\right) \ddot{A}=0 \\
& -\left(m_{\alpha \varepsilon}+j M_{\alpha \varepsilon}\right) \ddot{\varepsilon}-\left(c_{\alpha \varepsilon}+j C_{\alpha \varepsilon}\right) \dot{\varepsilon}-\left(k_{\alpha \varepsilon}+j K_{\alpha \varepsilon}-j K_{21}\right) \varepsilon \\
& +\left(K_{22}+K_{\alpha}-j k_{\alpha}\right) A+\left(C_{\alpha}-j c_{\alpha}-j \omega I_{p}\right) \dot{A}+\left(I_{d}+M_{\alpha}-j m_{\alpha}\right) \ddot{A}=0
\end{aligned}
$$

Considering the fundamental mode as shown in Fig. 2(a), we represent the displacements as follows.

$$
\left\{\begin{array}{l}
\varepsilon=X+j Y=\tilde{\varepsilon}\left(\varepsilon_{x}+j \varepsilon_{y}\right) \\
A=A_{x}+j A_{y}=\tilde{\alpha}\left(\alpha_{x}+j \alpha_{y}\right)
\end{array} ;\left\{\begin{array}{l}
\varepsilon_{x}=\cos (\Omega t) ; \varepsilon_{y}=\sin (\Omega t) \\
\alpha_{y}=-\cos (\Omega t) ; \alpha_{x}=\sin (\Omega t)
\end{array} ;\left\{\begin{array}{l}
\varepsilon_{x}+j \varepsilon_{y}=e^{j \Omega t} \\
\alpha_{x}+j \alpha_{y}=-j e^{j \Omega t}
\end{array}\right.\right.\right.
$$

Then we can express

$$
\left\{\begin{array} { l } 
{ \varepsilon = \tilde { \varepsilon } e ^ { j \Omega t } } \\
{ \dot { \varepsilon } = j \Omega \tilde { \varepsilon } e ^ { j \Omega t } } \\
{ \ddot { \varepsilon } = - \Omega ^ { 2 } \tilde { \varepsilon } e ^ { j \Omega t } }
\end{array} \left\{\begin{array}{l}
A=-j \tilde{\alpha} e^{j \Omega t} \\
\dot{A}=\Omega \tilde{\alpha} e^{j \Omega t} \\
\ddot{A}=j \Omega^{2} \tilde{\alpha} e^{j \Omega t}
\end{array}\right.\right.
$$

By substituting Eq. (17) into Eqs. (14) and (15), the following equations can be obtained:

$$
\begin{aligned}
& {\left[\left(M_{d}+M-j m\right)\left(-\Omega^{2}\right)+j \Omega(C-j c)+\left(K_{11}+K-j k\right)\right] \tilde{\varepsilon}} \\
& +\left[-j\left(k_{\varepsilon \alpha}+j K_{\varepsilon \alpha}-j K_{12}\right)+\Omega\left(c_{\varepsilon \alpha}+j C_{\varepsilon \alpha}\right)+j \Omega^{2}\left(m_{\varepsilon \alpha}+j M_{\varepsilon \alpha}\right)\right] \tilde{\alpha}=0 \\
& {\left[\left(m_{\alpha \varepsilon}+j M_{\alpha \varepsilon}\right) \Omega^{2}-j \Omega\left(c_{\alpha \varepsilon}+j C_{\alpha \varepsilon}\right)-\left(k_{\alpha \varepsilon}-j K_{\alpha \varepsilon}-j K_{21}\right)\right] \tilde{\varepsilon}} \\
& +\left[-j\left(K_{22}+K_{\alpha}-j k_{\alpha}\right)+\Omega\left(C_{\alpha}-j c_{\alpha}-j \omega I_{p}\right)+j \Omega^{2}\left(I_{d}+M_{\alpha}-j m_{\alpha}\right)\right] \tilde{\alpha}=0
\end{aligned}
$$

We define the following parameters.

$$
\left\{\begin{array}{l}
A_{1}=\left(M_{d}+M-j m\right)\left(-\Omega^{2}\right)+j \Omega(C-j c)+\left(K_{11}+K-j k\right) \\
A_{2}=-j\left(k_{\varepsilon \alpha}+j K_{\varepsilon \alpha}-j K_{12}\right)+\Omega\left(c_{\varepsilon \alpha}+j C_{\varepsilon \alpha}\right)+j \Omega^{2}\left(m_{\varepsilon \alpha}+j M_{\varepsilon \alpha}\right) \\
A_{3}=\left(m_{\alpha \varepsilon}+j M_{\alpha \varepsilon}\right) \Omega^{2}-j \Omega\left(c_{\alpha \varepsilon}+j C_{\alpha \varepsilon}\right)-\left(k_{\alpha \varepsilon}-j K_{\alpha \varepsilon}-j K_{21}\right) \\
A_{4}=-j\left(K_{22}+K_{\alpha}-j k_{\alpha}\right)+\Omega\left(C_{\alpha}-j c_{\alpha}-j \omega I_{p}\right)+j \Omega^{2}\left(I_{d}+M_{\alpha}-j m_{\alpha}\right)
\end{array}\right.
$$

Then equations (18) and (19) can be expressed as follows.

$$
\left[\begin{array}{ll}
A_{1} & A_{2} \\
A_{3} & A_{4}
\end{array}\right]\left[\begin{array}{l}
\tilde{\varepsilon} \\
\tilde{\alpha}
\end{array}\right]=0
$$

The condition for the linear homogeneous equation (21) to have nontrivial solution is

$$
\left|\begin{array}{cc}
A_{1} & A_{2} \\
A_{3} & A_{4}
\end{array}\right|=0
$$

Since Eq. (22) is a fourth order polynomial equation in terms of $\Omega$, we can obtain four complex roots of $\Omega$ for a cert ain rotational speed of the shaft $\omega$. Generally $\Omega$ is a complex value:

$$
\Omega=\Omega_{R}+j \Omega_{I}
$$

where the real part $\Omega_{R}$ represents the frequency, and the imaginary part $\Omega_{I}$ represents the damping rate.

\section{Results and Discussions}

Numerical calculations were made for the rotor shown in Fig. 3 used in the experiments to determine the fluid force moments. The numerical values of parameters are given in the Nomenclature. The case of $C_{2}=4 \mathrm{~mm}, v_{l} / U_{T}=0.170$, and $U_{J} / U_{T}=0$ are considered if not otherwise specified.

\subsection{The Effect of Fluid Force Moment on the Natural Frequency of the Rotor System}

Figure 8 shows the effect of each fluid force moment component on the complex frequency $\Omega=\Omega_{R}+j \Omega_{I}$ of the rotor-shaft system in the case of shaft length $L / D_{T}=3.344$. The amplitude grows exponentially if the imaginary part $\Omega_{I}$ is negative. Since we focus on the fluid force moments, the fluid forces and the gyroscopic moment are neglected. The horizontal axis is the rotational speed ratio $\omega / \omega_{n}$, where $\omega_{n}\left(=\frac{1}{2} \sqrt{\frac{K_{11}}{M_{d}}}=\sqrt{\frac{3 E I}{L^{3} M_{d}}}\right)$ denotes the natural frequency of the lateral vibration of the cantilevered rotorshaft system. The plot of the results up to large $\omega / \omega_{n}$ is just to show the vibration characteristics more clearly, the validity of the fluid force moments has not been confirmed at high speeds. $F_{1}, F_{2}$ denote the first and second-order forward vibrations with $\Omega_{R}>0$, respectively. $B_{1}, B_{2}$ denote the first and second-order of backward vibrations with $\Omega_{R}<0$, respectively. However, we identify "forward" or "backward" not from the sign on $\Omega_{R}$ at each $\omega / \omega_{n}$ but from the sign of $\Omega_{R}$ to which the solution approaches in the limit $\omega / \omega_{n} \rightarrow 0$.

For the case without applying the fluid force moments as shown in Fig. 8(a), the two sets of conjugate roots of Eq. (22) can be stated as below, 


$$
\Omega_{F_{2}, F_{1}, B_{2}, B_{1}}=\frac{ \pm \sqrt{2}}{2 I_{d} M_{d}} \sqrt{I_{d} M_{d}\left[I_{d} K_{11}+K_{22} M_{d} \pm \sqrt{\left(I_{d}^{2} K_{11}^{2}-2 I_{d} K_{11} K_{22} M_{d}+K_{22}^{2} M_{d}^{2}+4 I_{d} M_{d} K_{21} K_{12}\right)}\right]}
$$

where, $\Omega_{F_{1}}=-\Omega_{B_{1}}, \Omega_{F_{2}}=-\Omega_{B_{2}}$. The plus sign on $\sqrt{2}$ in Eq. (24) is taken for the "forward" modes $\Omega_{F_{1,2}}$ and the minus sign for the "backward" modes $\Omega_{B_{1,2}}$. The minus sign in the square root is taken for the "first order" modes $\Omega_{F, B_{1}}$ and the plus sign for the "second order" modes $\Omega_{F, B_{2}}$. The imaginary part of the frequency $\Omega$ is always 0 . It was confirmed that the numerical values obtained from the computations without fluid moment agree with Eq. (24).

Figure 8(b) shows the complex frequencies under the condition with the whirl fluid force moment. The value of $\Omega_{R}$ is different from that without fluid forces even at $\omega / \omega_{n}=0$ due to the fluid mass coefficients and changes with the rotational speed of the shaft $\omega / \omega_{n}$ caused by other fluid-dynamic parameters. The imaginary parts $\Omega_{I}$ of $F_{1}$ and $B_{1}$ are mainly negative showing that the whirl moment mostly destabilizes the whirling motion as expected from the results in Fig. 4(a). The second order component is stabilizing except for $F_{2}$ at smaller $\omega / \omega_{n}$. This is because the destabilization by the whirl moment occurs through the structural coupling as shown in [10] between linear $(\tilde{\varepsilon})$ and angular ( $\tilde{\alpha})$ displacements as shown in Fig. 12(b). It will be shown later that the 2nd order component includes only angular displacement as shown in Fig. 12(d).

Figure 8(c) shows the complex frequencies with the precession moment. It shows that all of the real parts of the frequencies $\Omega_{R} / \omega_{n}$ increase with increasing the rotational speed of the shaft. The real part of $B_{1}$ increases from negative to positive as we increase the rotational speed. Positive $\Omega_{I}$ of $B_{1}$ and negative $\Omega_{I}$ of $F_{1}$ in $\omega / \omega_{n}>2.0$ are expected from the results shown in Fig. 5(a), as will be discussed later.

Figure 8(d) shows the results with both whirl and precession moments. This result is similar to the result with only whirl moment shown in Fig. 8(b), suggesting that the effect of whirl moment is more important at the present shaft length of $L / D_{T}=3.344$.

\subsection{Correlation of Damping Rate $\Omega_{I}$ with the Normal Moment $M_{n}$}

In order to discuss about the destabilizing effect of the whirl and precession moments, the whirl speed ratio $\Omega_{R} / \omega$ and the damping rate $\Omega_{I} / \omega_{n}$ (in order to use the sign) are shown in Fig. 9(a) and (b), with whirl and precession moment, respectively. With the whirl moment, Fig. 9(a) shows that we have negative damping independently on the value of the whirl speed ratio $\omega / \omega_{n}$, since the value of $\Omega_{R} / \omega$ is outside of the region $0<\Omega_{R} / \omega<0.13$ where $m_{n_{-} W}$ in Fig. 4(a) becomes negative. This shows that the whirl moment is always destabilizing for the present case. On the other hand, Fig. 9(b) shows that the precession moment is stabilizing the backward mode $B_{1}$. The forward precession moment is stabilizing for the cases when $\Omega_{R} / \omega$ is larger than 0.52 but destabilizing

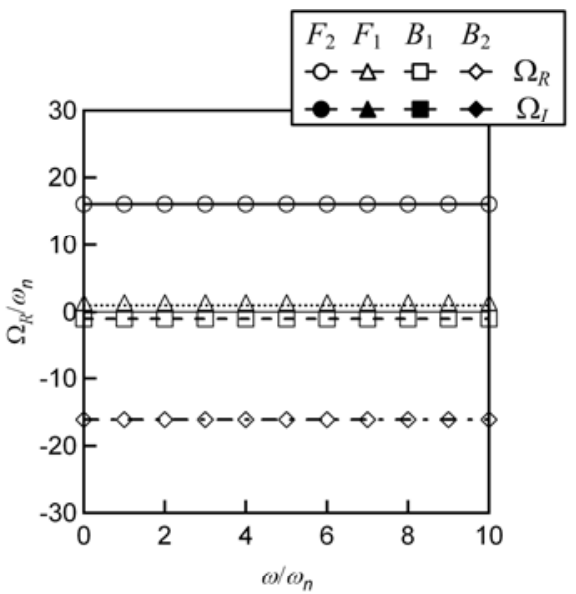

(a) Without fluid moment

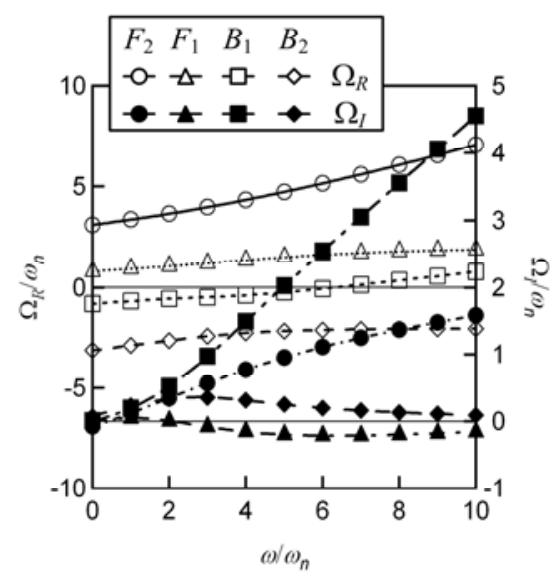

(c) With precession moment

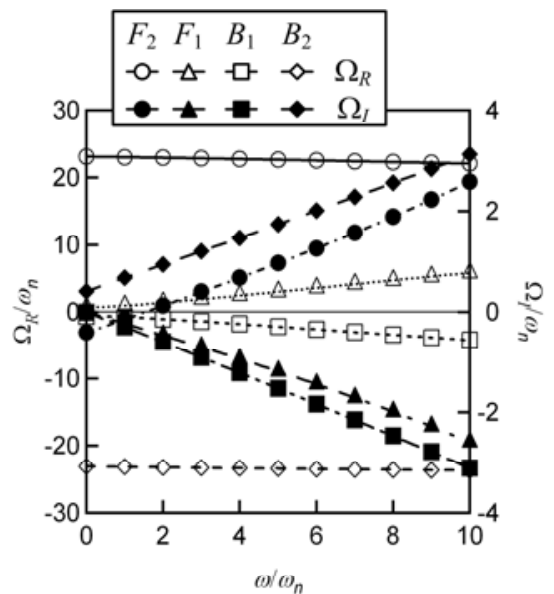

(b) With whirl moment

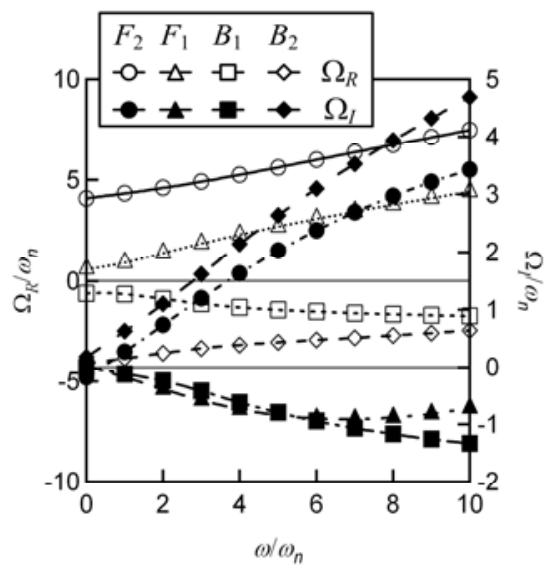

(d) With whirl and precession moments

Fig. 8 The effects of fluid force moments on the complex frequency of the rotor-shaft system, $L / D_{T}=3.344$, $C_{2}=4 \mathrm{~mm}, v_{l} / U_{T}=0.170, U_{J} / U_{T}=0$ 


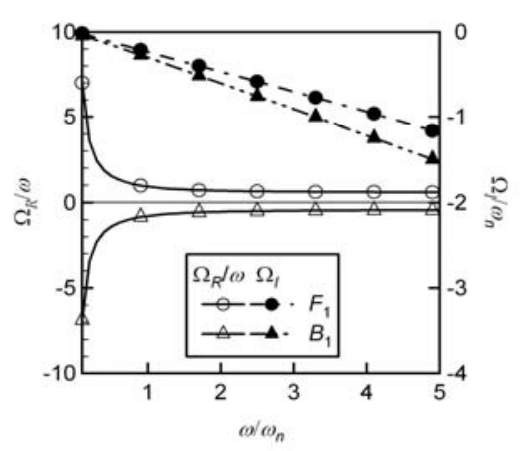

(a) With whirl moment

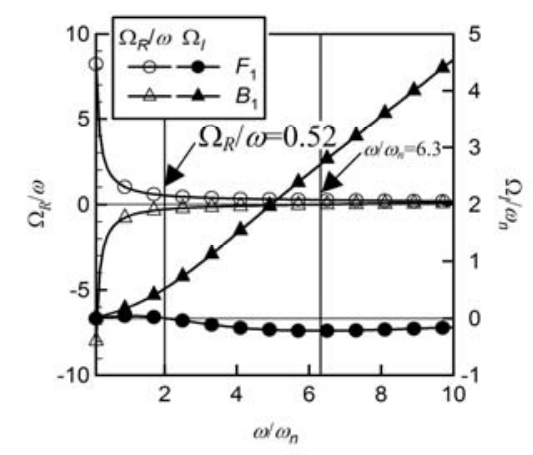

(b) With precession moment

Fig. 9 Fluid force moment effect on the first-order frequency of the rotor-shaft system, $L / D_{T}=3.344$ $C_{2}=4 \mathrm{~mm}, v_{/} / U_{T}=0.170, U_{J} / U_{T}=0$

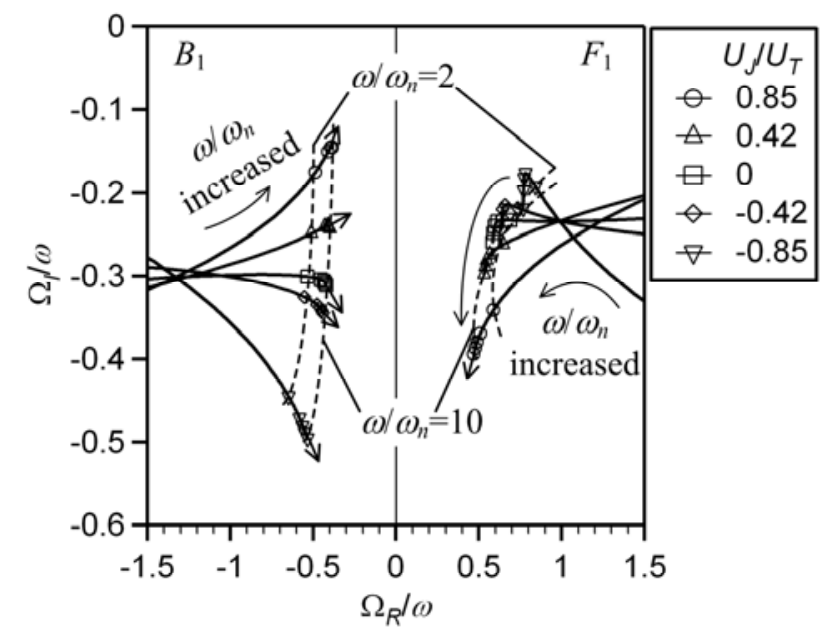

(a) With whirl moment

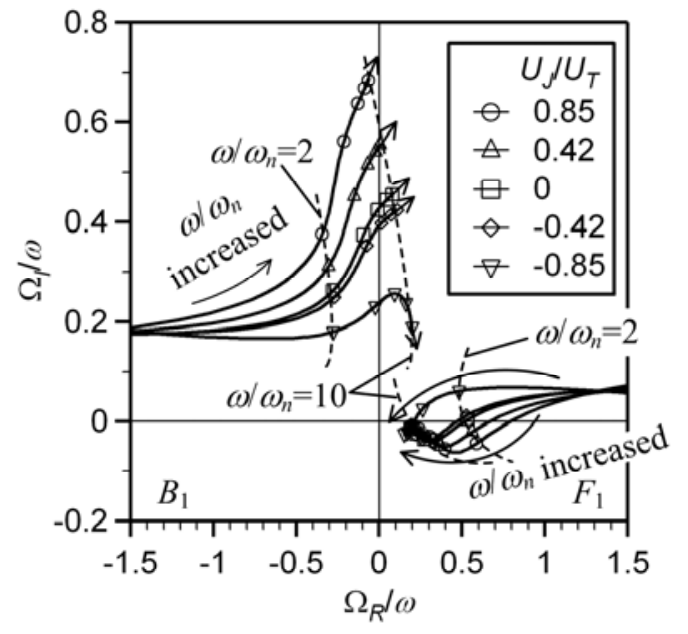

(b) With precession moment

Fig. 10 The swirl velocity effect on the roots locus of the rotor eigenvalues

when smaller than 0.52 . If we refer to the normal moment $m_{n_{-} w}$ and $m_{n_{-} p}$ shown in Fig. 4(a) and Fig. 5(a), we find that these results agree with the result of simplified stability analysis that "moments are destabilizing when the normal component of the moment has the same sign as the whirl/precession speed ratio". However, Fig. 9(b) shows that $\Omega_{R} / \omega$ of the backward mode $B_{1}$ becomes positive for $\omega / \omega_{n}>6.3$. In this region $m_{n_{-} p}$ shown in Fig. 5(a) is positive and $\Omega_{I} / \omega_{n}<0$ is expected from the simplified analysis. So, the criteria from the simplified analysis cannot be applied in this region.

Figure 10 shows the trace of damping coefficients $\Omega_{I} / \omega$ and the frequency coefficients $\Omega_{R} / \omega$, which corresponds to the angular velocity ratio $\Omega / \omega$ in the experiment, of the lowest frequency modes $F_{1}$ and $B_{1}$, as the rotor speed $\omega / \omega_{n}$ is increased for the case with $L / D_{T}=3.344$. The effect of inlet pre-swirl jet velocity $U_{J} / U_{T}$ is examined. The purpose of these plots is to examine the correlation of damping coefficients $\Omega_{I} / \omega$ with the normal fluid force moments $m_{n_{-} W}$ and $m_{n_{-} p}$. The arrow denotes the direction of the trace when $\omega / \omega_{n}$ is increased. The thick density of the plot around $\omega / \omega_{n}=10$ denote the solutions do not depend largely on $\omega / \omega_{n}$ when $\omega / \omega_{n}>2.0$.

Figure 10(a) shows the results with only the whirl moment. The negative value of $\Omega_{I} / \omega$ shows that the whirl moment always destabilizes the first order modes $F_{1}$ and $B_{1}$. First, we examine the backward mode $B_{1}$ in the range $-1<\Omega_{R} / \omega<0$. As the jet velocity increases, $\Omega_{I} / \omega$ increases correspondingly to the increase of the normal moment shown in Fig. 4(a). Second, we examine the forward mode $F_{1}$ in the range $0<\Omega_{R} / \omega<1$. As the jet velocity increases, the value of $\left|\Omega_{I} / \omega\right|$ increases according to the increase of the normal moment. Thus, the inlet swirl in the direction of rotation of the rotor has the effect to increase the destabilization of the forward whirl and to decrease the destabilization of the backward whirl.

Figure 10(b) shows the results with only the precession moment. In the region of backward precession $B_{1}$ with $\Omega_{R} / \omega<0$, the imaginary parts $\Omega_{I} / \omega$ are positive. This shows that the normal moment in the backward precession motion stabilizes the precession motion. The normal moment and hence $\Omega_{I} / \omega$ increases as the jet velocity increases. For the forward precession motion $F_{1}$, the normal moment destabilizes the precession motion in a region of small positive angular velocity ratio $\Omega_{R} / \omega$. The region changes largely as the jet velocity changes. Detailed examinations show that the value of $\Omega_{R} / \omega$ where $\Omega_{I} / \omega$ becomes zero in Fig. 10(b) agrees with the value of $\Omega_{R} / \omega$ where $m_{n_{-} P}$ becomes zero in Fig. 5(a).

\subsection{Effects of the Shaft Length on the Natural Frequency and Vibration Mode}

\subsubsection{For the case without fluid force moments}

We examine the effects of the shaft length $L$ on the natural frequencies given by Eq. (24) while keeping the values of other parameters to be constant.

Figure 11 shows the effect of the shaft length $L$ on the 1 st and the 2 nd order frequencies for the case without fluid force 


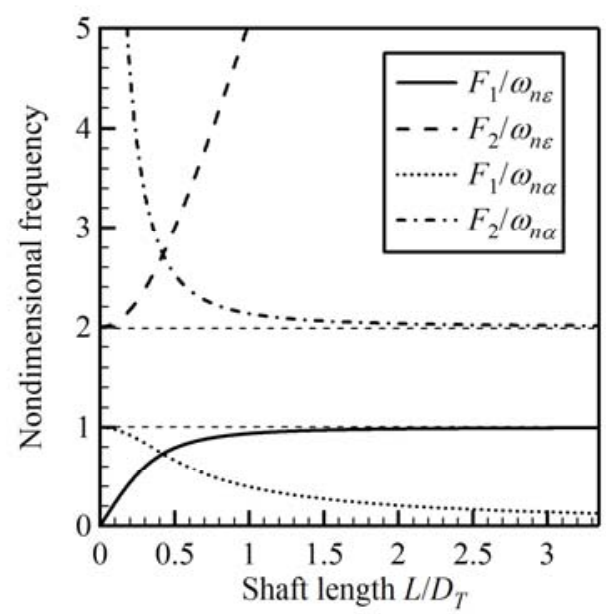

Fig. 11 The 1st and 2nd order frequencies for the case without fluid force moment

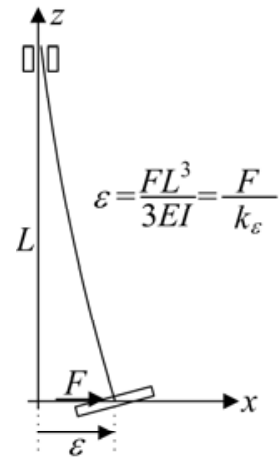

(a) $M=0$ (1st order with longer shaft)

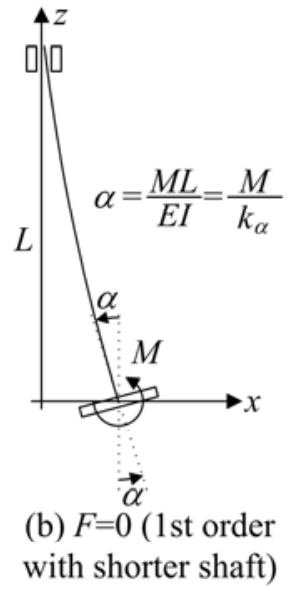

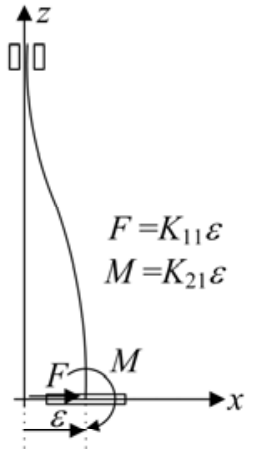

(c) $\alpha=0$ (2nd order with shorter shaft)

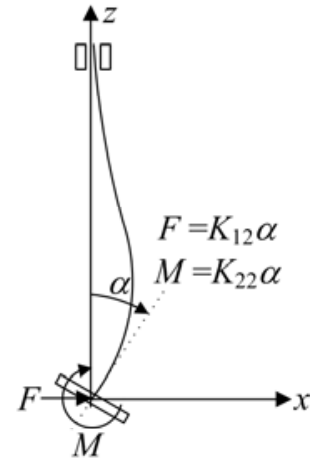

(d) $\varepsilon=0$ (2nd order with longer shaft)

Fig. 12 Mode of deflection and associated stiffness

moment. It can be shown from Eq. (24) that for shorter shafts with $\frac{L^{2}}{3} \cdot \frac{M_{d}}{I_{d}} \ll<1$, the 1 st order frequencies are $\Omega_{F_{1}}=-\Omega_{B_{1}} \approx \omega_{n \alpha}=\sqrt{\frac{k_{\alpha}}{I_{d}}}=\frac{1}{2} \sqrt{\frac{K_{22}}{I_{d}}}$ and the 2 nd order frequencies $\Omega_{F_{2}}=-\Omega_{B_{2}} \approx 2 \omega_{n \varepsilon}=\sqrt{\frac{K_{11}}{M_{d}}}$; for longer shafts with $\frac{L^{2}}{3} \cdot \frac{M_{d}}{I_{d}} \gg>1$, the first order frequencies $\Omega_{F_{1}}=-\Omega_{B_{1}} \approx \omega_{n \varepsilon}=\sqrt{\frac{k_{\varepsilon}}{M_{d}}}=\frac{1}{2} \sqrt{\frac{K_{11}}{M_{d}}}$ and the 2nd order frequencies $\Omega_{F_{2}}=-\Omega_{B_{2}} \approx 2 \omega_{n \alpha}=\sqrt{\frac{K_{22}}{I_{d}}}$, where, $k_{\varepsilon}=K_{11} / 4=3 E I / L^{3}$ and $k_{\alpha}=K_{22} / 4=E I / L . k_{\varepsilon}=F / \varepsilon$ is the stiffness showing the relation between the force $F$ and linear deflection $\varepsilon$, without applying the moment $M . k_{\alpha}=M / \alpha$ is the stiffness showing the relation between the moment $M$ and the angular displacement $\alpha$, without applying the force $F$. Equation (18) shows that $\tilde{\varepsilon} /(L \tilde{\alpha})=2 / 3$ when $\Omega=\omega_{n \varepsilon}=(1 / 2) \sqrt{K_{11} / M_{d}}$ and $\tilde{\alpha}=0$ when $\Omega=2 \omega_{n \varepsilon}=\sqrt{K_{11} / M_{d}}$. Equation (19) shows that $\tilde{\varepsilon} /(L \tilde{\alpha})=1 / 2$ when $\Omega=\omega_{n \alpha}=(1 / 2) \sqrt{K_{22} / I_{d}}$ and $\tilde{\varepsilon}=0$ when $\Omega=2 \omega_{n \alpha}=\sqrt{K_{22} / I_{d}}$. Equation (4) for steady deflection shows that the moment is zero when $\tilde{\varepsilon} /(L \tilde{\alpha})=2 / 3$ and the force is zero when $\tilde{\varepsilon} /(L \tilde{\alpha})=1 / 2$.

Figure 12 shows the mode of deflection and associated stiffness. The above discussion shows that, for shorter shafts, the 1st order vibration mode is as shown in Fig. 12(b) and the 2nd order mode is as shown in Fig. 12(c); for longer shafts, the 1st order vibration mode is as shown in Fig. 12(a) and the 2nd order mode is as shown in Fig. 12(d).

Figure 13 shows the amplitude ratio $|\tilde{\varepsilon} /(L \tilde{\alpha})|$ and the phase difference $\operatorname{Arg}\{\tilde{\varepsilon} / \tilde{\alpha}\}$ obtained from Eq. (21). In the limit of $L / D_{T} \rightarrow 0$, the amplitude ratio $|\tilde{\varepsilon} /(L \tilde{\alpha})|$ for $F_{1}$ approaches $1 / 2$; the amplitude ratio $|\tilde{\varepsilon} /(L \tilde{\alpha})|$ of $F_{2}$ approaches infinity, because the angular displacement $\tilde{\alpha}$ approaches to 0 , as shown in Fig. 12(c). For longer shafts $\left(L / D_{T} \rightarrow \infty\right)$, the amplitude approaches $2 / 3$ for $F_{1}$ and 0 for $F_{2}$ as expected. The phase difference $\operatorname{Arg}\{\tilde{\varepsilon} / \tilde{\alpha}\}$ is always zero for $F_{1}$ and $\pi$ for $F_{2}$.

\subsubsection{For the case with fluid force moments}




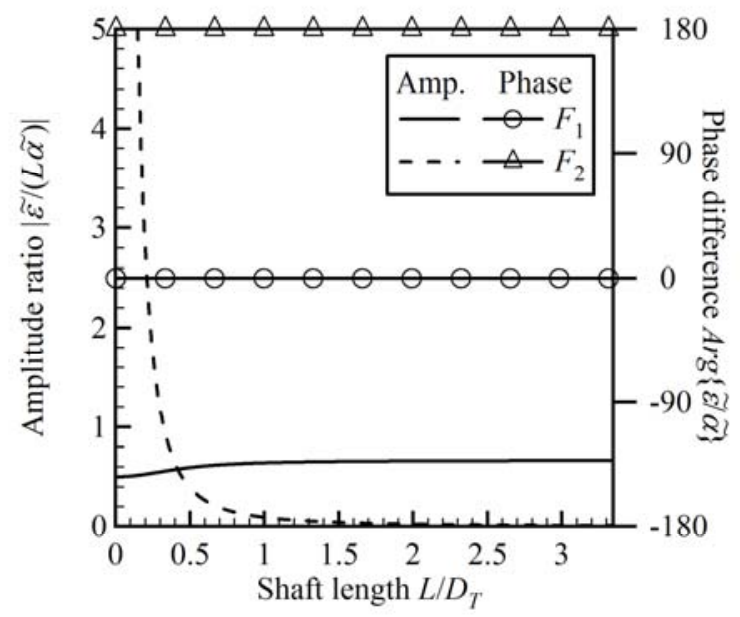

Fig. 13 Amplitude ratio $|\widetilde{\varepsilon}(L \tilde{\alpha})|$ and phase difference $\operatorname{Arg}\{\widetilde{\varepsilon} / \widetilde{\alpha}\}$ for the case without fluid force moment

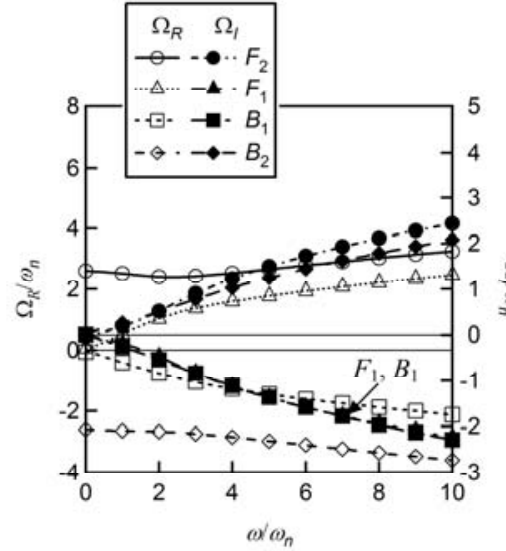

(a) With whirl moment

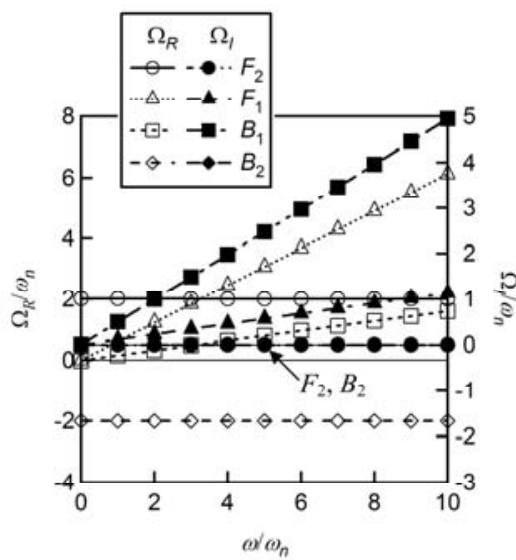

(b) With precession moment

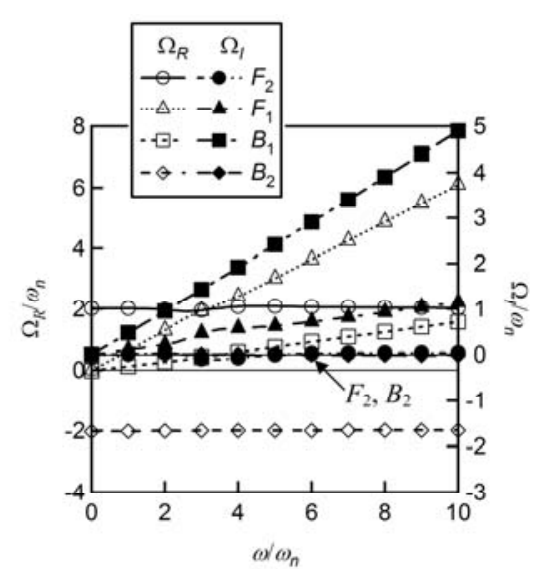

(c) With whirl and precession moments

Fig. 14 The complex frequency in the case of shaft length $L / D_{T}=0.033$

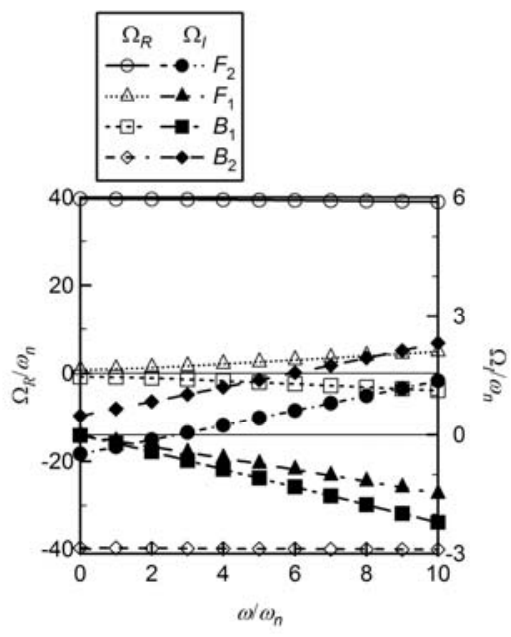

(a) With whirl moment

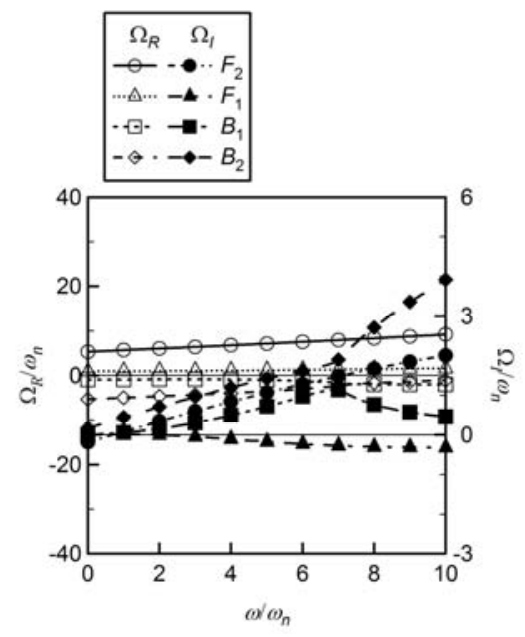

(b) With precession moment

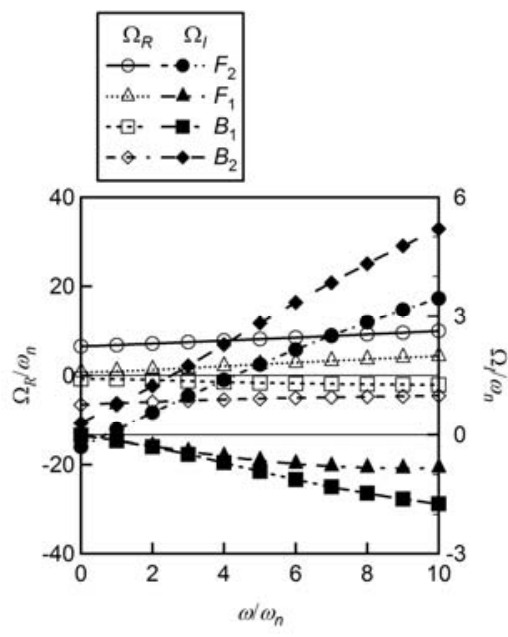

(c) With whirl and precession moments

Fig. 15 The complex frequency in the case of shaft length $L / D_{T}=6.688$

Figures 14 and 15 show the complex frequencies $\left(\Omega_{R} / \omega_{n}, \Omega_{I} / \omega_{n}\right)$ in the case of shaft length $L / D_{T}=0.033$ and 6.688 , respectively. Unrealistically extreme cases are considered here to clearly show the contributions of the whirl and precession moments. In Figs. 14 and 15, (a), (b) and (c) show the results with the whirl, precession, and both of the moments, respectively.

In the case of shorter shaft, shown in Fig. 14, both of the real part $\Omega_{R} / \omega_{n}$ and the imaginary part $\Omega_{I} / \omega_{n}$ shown in (c) are similar to the results shown in (b). This shows that the precession moment is important for the case of shorter shaft. On the other hand, the results shown in Fig. 15(c) are similar to the results shown in Fig. 15(a), which shows that the whirl moment is more important for the case of longer shafts. Firstly, we focus on the results of the first order frequency components $F_{1}$ and $B_{1}$. The whirl moments are always destabilizing the motion for both of shorter and longer shafts shown in Figs. 14(a) and 15(a). The precession moment 
destabilizes $F_{1}$ for $\omega / \omega_{n}>2$ for the longer shafts, shown in Fig. 15(b). This is the same as in Fig. 8(c) and Fig. 9(b). However, for $F_{1}$ for shorter shaft shown in Fig. 14(b), the precession moment stabilizes the vibration; this is because the angular velocity ratio $\Omega_{R} / \omega(\approx 0.62)$ is always larger than 0.5 for all the rotational speed ratio $0<\omega / \omega_{n}<10$. For the backward precession motion $B_{1}$, the frequency $\Omega_{R} / \omega_{n}$ is negative at $\omega / \omega_{n}=0$. However, for the case of shorter shaft with $L / D_{T}=0.033$ (Fig. 14(b)) and 3.344 (Fig. 8(c)), $\Omega_{R} / \omega_{n}$ increases with the increase of $\omega / \omega_{n}$ and becomes positive at higher values of $\omega / \omega_{n}$. For the case of Fig. 14(b), the value of $\Omega_{R} / \omega$ is approximately 0.16 for $B_{1}$ mode. Figure 5(a) shows that the normal moment is positive at $\Omega / \omega=0.16$. The result of $\Omega_{I}>0$ for $B_{1}$ shown in Fig. 14(b) contradicts to the result of the simplified stability analysis that "moments are destabilizing when the normal component of the moment has the same sign as the whirl/precession speed ratio". Similar discrepancy is found also for $B_{1}$ at larger value of $\omega / \omega_{n}$ shown in Fig. 8(c), where $\Omega_{R} / \omega_{n}$ becomes positive.

Secondly, we focus on the results of the second order frequency components $F_{2}$ and $B_{2}$. The second order components are basically stabilizing or neutrally stabilizing. With the whirl moment, this is reasonable since the destabilization by the whirl moment is through the structural coupling of whirl and precession motion. Figure 14(b) shows that the precession moment is near neutral stability with small values of $\Omega_{I} / \omega_{n}$. However, it includes a destabilizing region. Figure 18 shows the plot of $\Omega_{I} / \omega$ against $\Omega_{R} / \omega$ with $\omega / \omega_{n}$ as a parameter. The value of $\Omega_{I} / \omega$ becomes negative in the region $0<\Omega_{R} / \omega<0.5$ where Fig. 5(a) shows that $m_{n \_p}>0$ suggesting that the precession moment can destabilize the second order forward mode $F_{2}$.

Corresponding to the complex frequencies shown in Figs. 14 and 15, the amplitude ratio $|L \tilde{\alpha} / \tilde{\varepsilon}|$ and the phase difference $\operatorname{Arg}\{\tilde{\alpha} / \tilde{\varepsilon}\}$ of the vibration mode in the case of shaft length $L / D_{T}=0.033$ and 6.688 are shown in Figs. 16 and 17 , respectively. We first focus on the first order vibration mode. Near $\omega / \omega_{n}=0$, the amplitudes ratio $|L \tilde{\alpha} / \tilde{\varepsilon}|$ is kept nearly constant at 3/2 for longer shaft and 2 for shorter shaft, corresponding to the natural modes shown in Fig. 12(a) and (b) respectively. However it deviates from those values above $\omega / \omega_{n} \approx 2$. The phase difference is near zero in this region. Nearly the same order of $|L \tilde{\alpha} / \tilde{\varepsilon}|$ for the shorter and longer shafts means that the amplitude $\tilde{\varepsilon}$ is practically larger for longer shafts. In the same way as for the frequency, the amplitude ratio and the phase difference with both whirl and precession moments are similar to the case with only precessing moment for shorter shaft, and to the case with only whirl moment for the case of longer shaft. Next, we consider the second order components. The amplitude ratio $|L \tilde{\alpha} / \tilde{\varepsilon}|$ is near zero for shorter shafts and has larger values for longer shafts except for the result shown in Fig. 16(a). The phase difference is near $\pm \pi$ around $\omega / \omega_{n}=0$. It is interesting to note that the phase difference crosses 0 or $\pm \pi$ when $\Omega_{I}$ in Figs.14 and 15 becomes 0 . We can derive from Eq. (18)

$$
\tilde{\alpha} / \tilde{\varepsilon}=\frac{K_{11}-M_{d} \Omega^{2}}{K_{12}}=\frac{2}{L}\left[1-\frac{M_{d}}{K_{11}}\left(\Omega_{R}^{2}-\Omega_{I}^{2}\right)\right]-\frac{4}{L} \frac{M_{d}}{K_{11}} \Omega_{R} \Omega_{I} j
$$

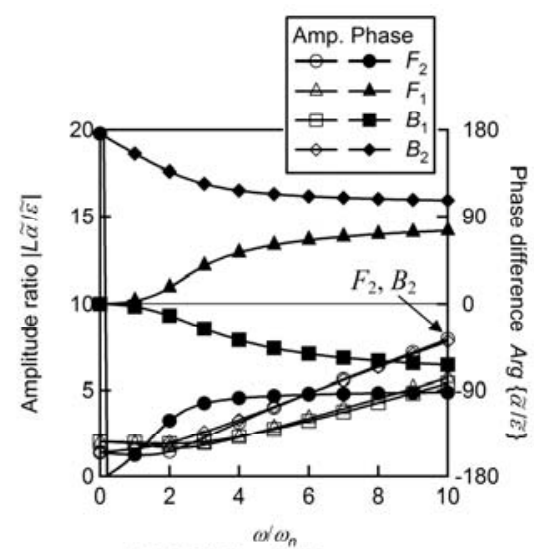

(a) With whirl moment

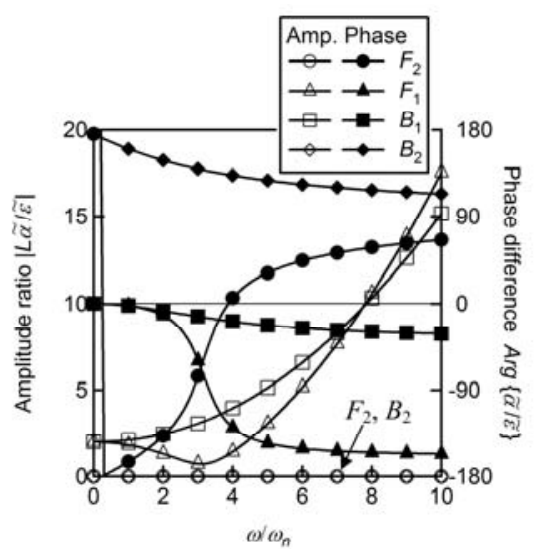

(b) With precession moment

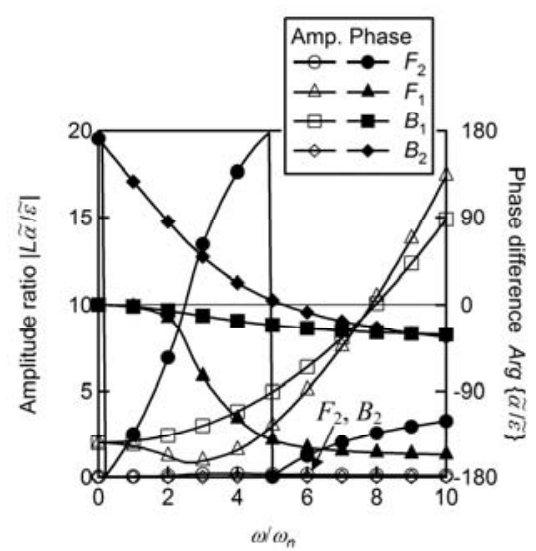

(c) With whirl and precession moments

Fig. 16 Amplitude ratio $|L \tilde{\alpha} / \widetilde{\varepsilon}|$ and phase difference $\operatorname{Arg}\{\tilde{\alpha} / \widetilde{\varepsilon}\}$ in the case of shaft length $L / D_{T}=0.033$

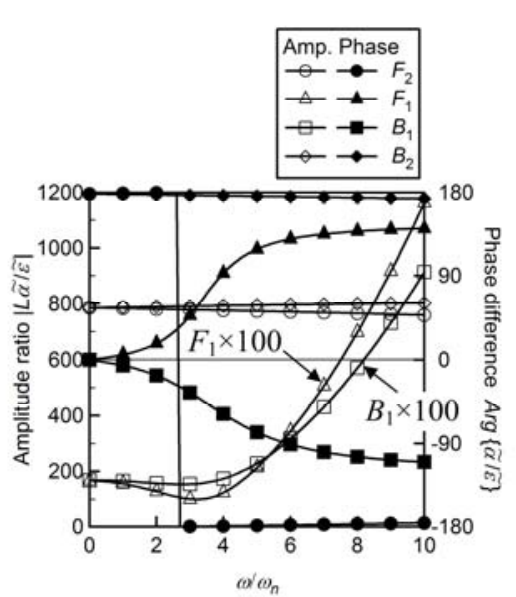

(a) With whirl moment

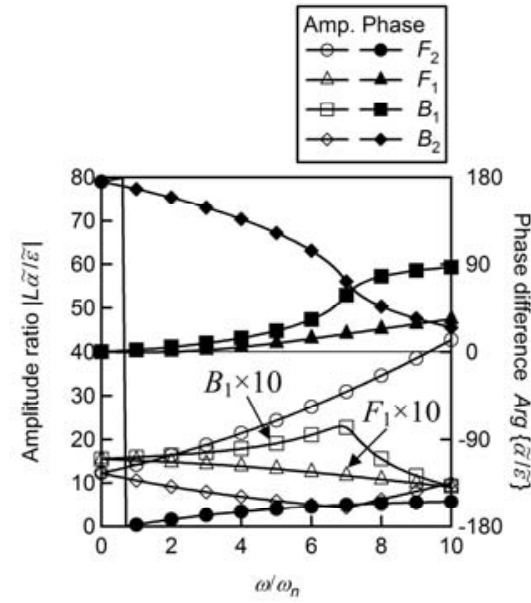

(b) With precession moment

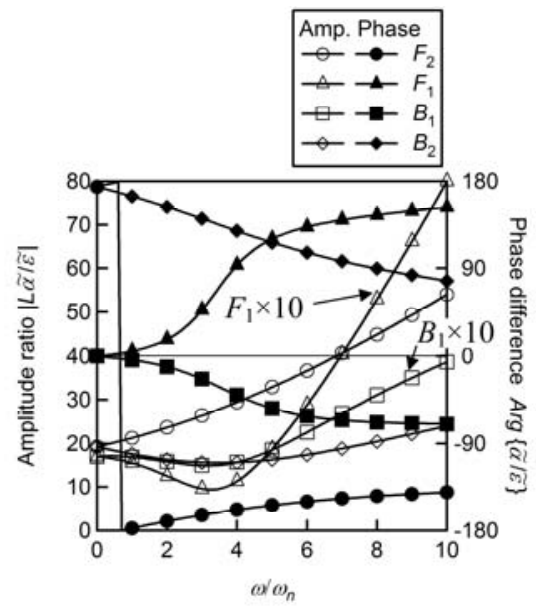

(c) With whirl and precession moments

Fig. 17 Amplitude ratio $|L \tilde{\alpha} / \widetilde{\varepsilon}|$ and phase difference $\operatorname{Arg}\{\widetilde{\alpha} / \widetilde{\varepsilon}\}$ in the case of shaft length $L / D_{T}=6.688$ 


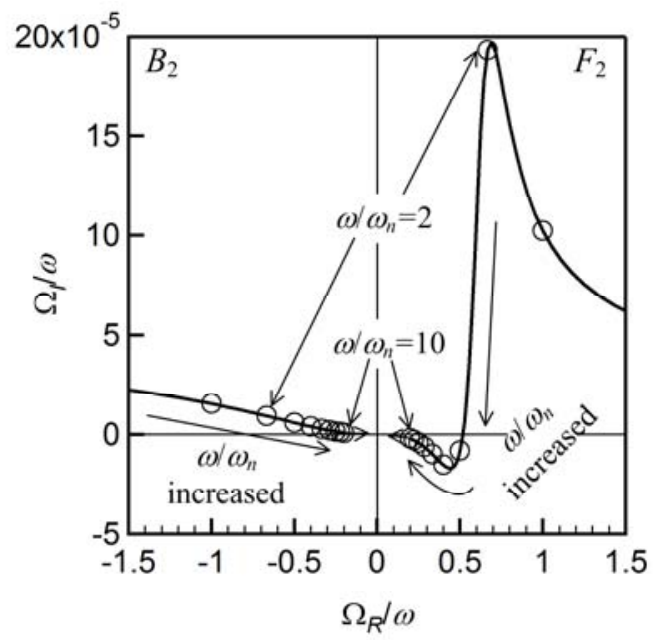

Fig. 18 Plot of $\Omega_{R} / \omega$ and $\Omega_{l} / \omega$ for the second order precession modes $\left(F_{2}, B_{2}\right), L / D_{T}=0.033$, with precession moment

by neglecting the fluid moment coefficients and using Eq. (5). The relation between the phase difference and the sign of $\Omega_{I}$ was confirmed for all cases with the effect of fluid moment.

In summary, it was found that the whirl moment is always destabilizing the vibration for the cases of both shorter and longer shafts. However, with shorter shaft, stabilizing effect of precession moment is stronger than the destabilizing effect of the whirl moment and suppresses the instability. Thus, we can conclude that the whirl moment is more important than the precession moment, for the destabilization of the first order mode of the overhung rotor.

In the above calculations, there are certain cases when the stability criteria obtained from simplified analysis cannot be applied. The simplified analysis assumes that the fluid force moments are so small as compared with other forces and the vibration mode is not largely affected by the fluid force moments. So, we carried out calculations with the fluid force moments reduced by a factor of 1000. Then we found that the destabilization by the whirl moment occurs in the region except for $0<\Omega_{R} / \omega<0.13$, and the destabilization by the precession moment in $0<\Omega_{R} / \omega<0.52$ for both longer and shorter shafts of the first order modes. This completely agrees with the stability criteria from simplified analysis and shows that the disagreement is caused by larger fluid force moments used for calculations.

\section{Conclusions}

The stability of an overhung rotor with the fluid force moment on a rotor caused by the leakage flow was examined based on a lumped parameter vibration model. The whirl and precession moments are considered. The results can be summarized as follows.

(1) The whirl moment destabilizes the first order modes of overhung rotors with shorter and longer shafts at all rotor speed.

(2) The precessing moment destabilizes the first order forward mode with longer shafts and the second order forward mode with shorter shaft in the range $0<\Omega_{R} / \omega<0.52$. However, it has stabilizing effects in other cases and is not important in the destabilization of overhung rotors.

(3) The whirl moment is more important for overhung rotors with longer shafts and the precessing moment is important for overhung rotors with shorter shafts.

(4) The second order modes are mostly stabilized by the whirl and precessing moments.

(5) The stability criteria obtained by a simplified analysis that "positive normal moment with positive whirl/precession and negative normal moment with negative whirl/precession have the effect to destabilize the vibration" can be applied for the most cases. However it cannot be applied to the cases when the backward mode becomes forward caused by larger fluid moments compared with structural moments.

\section{Acknowledgement}

Bingwei Song would like to thank China Scholarship Council for their financial support during his stay in Osaka University for carrying out this research.

\section{Nomenclature}

$\begin{array}{llll}A_{x}, A_{y} & \text { Angular displacements about } x \text { and } y \text { axis } & R_{T} & \text { Disk radius }(=0.1495[\mathrm{~m}]) \\ C_{1}, C_{2} & \text { Radial and axial clearances } & t & \text { Time or tangential direction } \\ D_{T} & \text { Diameter of the disk }(=0.299[\mathrm{~m}]) & U_{J} & \text { Jet velocity from the swirl generator } \\ E & \text { Young's modulus }\left(=2.0 \times 10^{11}[\mathrm{~Pa}]\right) & U_{T} & \text { Tip velocity of the disk }\left(=R_{T} \omega\right) \\ f & \text { Angular velocity ratio in experiment }(=\Omega / \omega) & v_{l} & \text { Leakage flow velocity }\left(=Q /\left(\pi D_{T} C_{1}\right)\right) \\ F_{x}, F_{y} & \text { External forces in } x \text { and } y \text { directions } & X, Y & \text { Linear displacements in } x \text { and } y \text { directions } \\ I & \text { Moment of inertia of the cross-section of the } & |\alpha| & \text { Angular displacement of the disk }(=0.48[\mathrm{deg}]) \\ & \begin{array}{l}\text { shaft }\left(=7.85 \times 10^{-9}\left[\mathrm{~m}^{4}\right]\right), \text { shaft diameter } \\ (=0.02[\mathrm{~m}])\end{array} & \tilde{\alpha} & \text { in the experiment } \\ & & \text { Amplitude of angular displacement of the }\end{array}$




\begin{tabular}{|c|c|c|c|}
\hline$I_{d}, I_{p}$ & Diametral and polar moment of inertia & & disk \\
\hline & $\left(I_{d}=0.011\left[\mathrm{Kg} \cdot \mathrm{m}^{2}\right], I_{p}=0.021\left[\mathrm{Kg} \cdot \mathrm{m}^{2}\right]\right)$ & $|\varepsilon|$ & Linear displacement of the disk $(=0.5[\mathrm{~mm}])$ \\
\hline$j$ & Imaginary unit & & in the experiment \\
\hline$L$ & Length of the shaft, $(=0.01 ; 1 ; 2[\mathrm{~m}])$ & $\tilde{\varepsilon}$ & Amplitude of linear displacement of the disk \\
\hline$M_{d}$ & Mass of the disk $(=2.079[\mathrm{Kg}])$ & $\phi$ & Phase difference of $\tilde{\alpha} / \tilde{\varepsilon}$ \\
\hline$M_{n}, M_{t}$ & Normal and tangential fluid force moments & $\rho$ & Density of working fluid $\left(=997.07\left[\mathrm{Kg} / \mathrm{m}^{3}\right]\right.$ \\
\hline$M_{n \_P}, M_{t \_}$ & $\begin{array}{l}\text { Normal and tangential precession fluid force } \\
\text { moments }\end{array}$ & $\Omega$ & $\begin{array}{l}\text { Angular velocity of whirling or precession } \\
\text { motion in experiment; Complex angular }\end{array}$ \\
\hline$m_{n \_}, m_{t \_} P$ & Normalized normal and tangential precession & & velocity in calculation \\
\hline$M_{n, W}, M_{t W}$ & fluid force moments & $\Omega_{R}$ & Real part of $\Omega$ in calculation \\
\hline$M_{n_{-} W}, M_{t_{-} W}$ & $\begin{array}{l}\text { mormal and tangential whirl iluid force } \\
\text { moments }\end{array}$ & $\Omega_{I}$ & $\begin{array}{l}\text { Imaginary part of } \Omega \text { in calculation } \\
\text { Rotational angular velocity of the shaft }\end{array}$ \\
\hline$m_{n \_W}, m_{t \_W}$ & $\begin{array}{l}\text { Normalized normal and tangential whirl } \\
\text { fluid force moments }\end{array}$ & $\begin{array}{l}\omega \\
\text { Superscript }\end{array}$ & 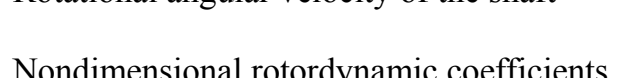 \\
\hline$M_{x}, M_{y}$ & External moments in $x$ and $y$ directions & & 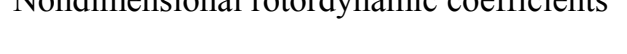 \\
\hline & Normal direction & Subscr1 & Fluid \\
\hline & Volume flow rate & & Gyro \\
\hline$R_{\text {inner }}$ & Inner radius of the casing $(=0.0475[\mathrm{~m}])$ & & \\
\hline
\end{tabular}

\section{References}

[1] Guinzburg, A., Brennen, C. E., Acosta, A. J., and Caughey, T. K., 1994, "Experimental Results for the Rotordynam ic Characteristics of Leakage Flows in Centrifugal Pumps," ASME Journal of Fluids Engineering, Vol. 116, pp. 110-115. [2] Uy, R., V., Brennen, C., E., 1999, "Experimental Measurements of Rotordynamic Forces Caused by Front Shroud Pump Leakage," ASME Journal of Fluids Engineering, Vol. 121, pp. 633-637.

[3] Hsu, Y., Brennen, C., E., 2002, "Effect of Swirl on Rotordynamic Forces Caused by Front Shroud Pump Leakage,” ASME Journal of Fluids Engineering, Vol. 124, pp. 1005-1010.

[4] Tsujimoto, Y., Yoshida, Y., Ohashi, H., Teramoto, N., and Ishizaki, S., 1997, "Fluid Force Moment on a Centrifugal Impeller Shroud in Precessing Motion," ASME Journal of Fluids Engineering, Vol. 119, pp. 366-371.

[5] Yoshida, Y., Tsujimoto, Y., Ohashi, H., Saito, A., and Ishizaki, S., 1997, "Measurements of the Flow in Backshroud/Casing Clearance of Precessing Centrifugal Impeller," International Journal of Rotating Machinery, Vol. 3, No. 4, pp. $259-268$.

[6] Tomita, H. and Kawamura, M., 1965, "Self-Excited Vibration in Francis Turbines," Toshiba Review, Vol.20, No. 8, pp. 787791, (in Japanese).

[7] Ohashi, H., 1991, Vibration and Oscillation of Hydraulic Machinery (International Hydraulic machinery Series), Avebury Technical, Aldershot.

[8] Kanemori, Y. and Iwatsubo, T., 1992, "Experimental Study of the Dynamic Fluid Forces and Moments for a Long Annular Seal," ASME Journal of Tribology, Vol. 114, pp.773-778.

[9] Kanemori, Y. and Iwatsubo, T., 1994, "Forces and Moments Due to Combined Motion of Conical and Cylindrical Whirls for a Long Seal," ASME Journal of Tribology, Vol. 116, pp. 489-498.

[10] Song, B., Horiguchi, H., Ma, Z., Tsujimoto, Y., 2009, "Rotordynamic Fluid Force Moment on the Backshroud of a Francis Turbine Runner under Whirling Motion," Turbomachinery, Vol. 3, No. 10, pp. 50-61 (in Japanese).

[11] Song, B., Horiguchi, H., Nishiyama, Y., Hata, S., Ma, Z., Tsujimoto, Y., 2009, "Fluid Force Moment on the Backshroud of a Francis Turbine Runner in Precession Motion," Proceeding of the 62nd Turbomachinery Society of Japan Conference, pp. 7-12.

[12] Childs, D., W., 1989, "Fluid-Structure Interaction Forces at Pump-Impeller-Shroud Surfaces for Rotor Dynamics Calculation," ASME Journal of Vibration, Acoustics, Stress and Reliability in Design, Vol. 111, pp. 216-225.

[13] Childs, D., W., 1993, “Turbomachinery Rotordynamics: Phenomena, Modeling, \& Analysis,” John Wiley \& Sons, New York. 